\title{
Chronic Pseudophakic Aqueous Misdirection
}

\author{
Mona A. Kaleem ${ }^{1,2}$, Sheldon Oberfeld ${ }^{1}$, Jonathan Eisengart ${ }^{1}$ \\ ${ }^{1}$ Cole Eye Institute, Cleveland Clinic, Cleveland, Ohio, USA; ${ }^{2}$ Department of \\ Ophthalmology, University of Maryland, Baltimore, Maryland, USA
}

\begin{abstract}
A 61 year old female with no prior ocular history developed progressive anterior chamber shallowing following uncomplicated phacoemulsification and intraocular lens implantation. This shallowing rapidly accelerated after Nd:YAG laser capsulotomy, and led to a significant myopic shift. Ultrasound biomicroscopy imaging demonstrated anterior displacement of the irido-lenticular diaphragm and anterior rotation of ciliary processes confirming a diagnosis of aqueous misdirection. One year after Nd:YAG anterior hyaloidotomy and medical therapy, her anterior chamber deepened and myopic shift resolved. This case demonstrates the successful management of chronic pseudophakic aqueous misdirection without the need for surgical intervention.
\end{abstract}

Key words: aqueous misdirection, malignant glaucoma, Nd:YAG capsulotomy

\section{Introduction}

Aqueous misdirection syndrome, also known as malignant glaucoma, is a diagnosis of exclusion characterized by anterior chamber (AC) shallowing and often elevated intraocular pressure (IOP) in the absence of pupillary block or choroidal hemorrhage. It is an uncommon complication of intraocular surgery, laser therapy, or use of miotic agents. Aqueous misdirection is usually associated with a history of angle closure glaucoma or short axial length. It is most commonly precipitated by glaucoma filtering surgery, with more than $75 \%$ of cases occurring after trabeculectomy and $3.7-2.8 \%$ after a tube shunt procedure. ${ }^{1}$ There have been a few reports of aqueous misdirection after intra or extra-capsular cataract surgery with an estimated incidence between $0.03 \%-0.025 \%$, but there is no data from the era of phacoemulsification surgery. ${ }^{1,2}$ The incidence of misdirection after Nd:YAG capsulotomy is unknown and only a few case reports exist in the literature. ${ }^{3}$

Here we report a case of chronic aqueous misdirection presenting with $\mathrm{AC}$ shallowing, normal IOP, and a significant myopic shift six months after phacoemulsification and two weeks after Nd:YAG laser capsulotomy. The features of this case demonstrate the subtle clinical signs and symptoms phacoemulsification surgeons should take into account when evaluating patients in the post-operative period.

\section{Case Report}

A 61 year old black female with mature nuclear sclerosis and posterior subcapsular cataracts underwent uncomplicated phacoemulsification cataract extraction and posterior chamber intraocular lens implantation in both eyes. She had no prior

Correspondence: Jonathan Eisengart, Cole Eye Institute, Cleveland Clinic, 2022 E 105th

St, Cleveland, $\mathrm{OH} 44106$, USA

E-mail: eisengj@ccf.org 
ocular history to report; her pre-operative anterior segment exam was normal with the exception of mature cataract. Posterior segment exam was limited by brunescent cataract but a symmetrical red reflex was noted. IOP was $10 \mathrm{~mm} \mathrm{Hg}$. Pre-operative refraction was difficult due to density of the cataract, however, was measured as: OD: $-4.75+3.25 \times 105$ correcting to hand motion and OS: $-1.25+3.00$ $x 081$ correcting to count finger at one foot. Axial length as measured by A-scan was OD: $20.95 \mathrm{~mm}$ and OS: $20.80 \mathrm{~mm}$. Phacoemulsification and intraocular lens implantation into the capsular bag was performed in the right eye first with no ensuing events to report, followed by the left eye two months later.

An Alcon SN60WF 27.5 D lens was placed in the left eye with a target refraction of $-0.91 \mathrm{D}$. Her post-operative course was uneventful although the surgeon noted the $A C$ of the left eye was slightly shallower than the right eye. IOP ranged from $10-18 \mathrm{~mm} \mathrm{Hg}$. At post-op month one her refraction was stable at $-1.50 \mathrm{D}$ correcting to $20 / 30$.

She returned for her six month follow up exam complaining of blurry vision. Her refraction measured $-2.25+0.50 \times 085$ correcting to $20 / 40$ and she underwent $\mathrm{Nd}$ :YAG laser capsulotomy for posterior capsular opacification. Two weeks after laser, her refraction changed to $-7.25+4.50 \times 090$ correcting to $20 / 25$. On slit lamp she was noted to have moderate $A C$ shallowing. The lens implant remained securely within the capsular bag in proper orientation with an open posterior capsule. IOP was measured as $17 \mathrm{~mm} \mathrm{Hg}$ and gonioscopy revealed angle closure with peripheral anterior synchiae in three quadrants, and one quadrant with appositional closure. The fellow eye was wide open to ciliary body band. The UBM image in figure 1 shows angle configuration of the unaffected right eye with AC depth of $3.65 \mathrm{~mm}$. Figures 2 and 3 of the left eye demonstrate an AC depth of $2.42 \mathrm{~mm}(1.23 \mathrm{~mm}$ of shallowing of the left eye AC compared to the right eye), anterior displacement of the IOL-iris diaphragm, and anterior rotation of ciliary processes. There was no iris bombe configuration to suggest pupillary block.

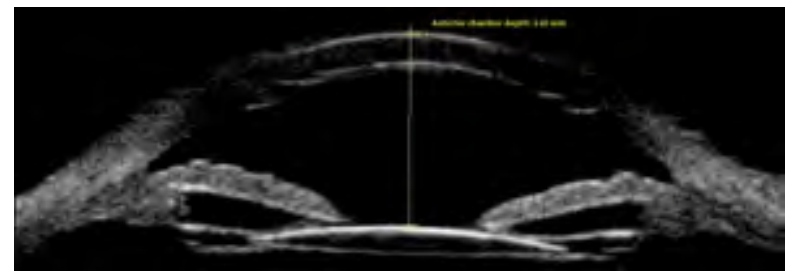

Fig 1. UBM image of the right eye on initial presentation. Anterior chamber depth measured as $3.65 \mathrm{~mm}$.

Her posterior segment exam was normal. There was no evidence of choroidal effusion or hemorrhage on a careful peripheral exam, nor was there evidence of a supraciliary effusion on UBM. Because the anterior hyaloid was still visibly intact at the slit lamp, an Nd:YAG anterior hyaloidotomy was performed using $2.3-3.5 \mathrm{~mJ}$. Immediately afterward her AC deepened to $2.92 \mathrm{~mm}$. She was started on atropine $1 \%$ and timolol $0.5 \%$.

At one month follow up on topical therapy, her refraction returned to -1.50 correcting to $20 / 30$ and her IOP remained within normal limits at $18 \mathrm{~mm} \mathrm{Hg}$. Her AC 
maintained the post anterior hyaloidotomy configuration which was deeper than prior to treatment, but still shallower than the fellow eye. Atropine and timolol were stopped.
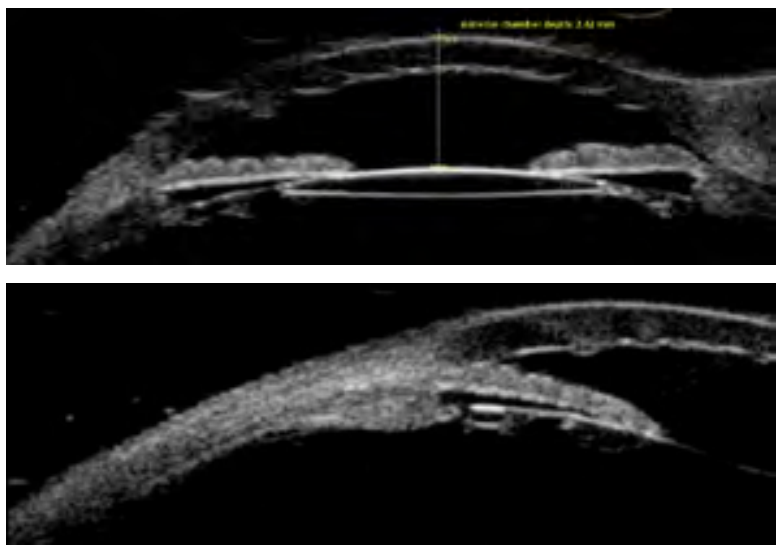

Fig 2 \& 3. UBM images of the left eye on initial presentation. Anterior chamber depth measured as $2.42 \mathrm{~mm}$.

Six months after stopping topical therapy she had a mild recurrence of $A C$ shallowing and anterior displacement of the lens to iris diaphragm with an $A C$ depth of $2.72 \mathrm{~mm}$ as documented on UBM imaging, a myopic shift, and IOP of $19 \mathrm{~mm} \mathrm{Hg}$. On gonioscopy, she was noted to have angle closure with peripheral anterior synechiae in all four quadrants. Atropine and timolol eye drops were reinstituted for several months. Her AC returned to a normal depth of $3.47 \mathrm{~mm} \mathrm{Hg}$ (only $0.18 \mathrm{~mm}$ shallower than the right eye) with resolution of myopic shift and aqueous misdirection (Figure 4).

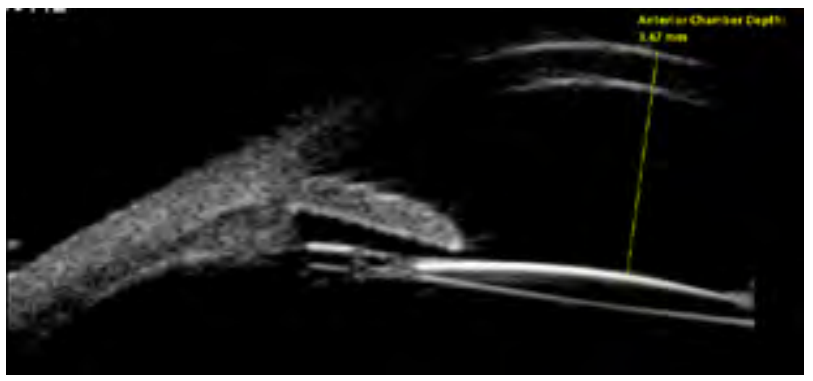

Fig 4. UBM image of the left eye one year after following Nd:YAG laser anterior hyaloidotomy on medical therapy. Anterior chamber depth measured as $3.47 \mathrm{~mm}$.

\section{Discussion}

Aqueous misdirection was first described by Dr. von Graefe as an early post-surgical complication in patients with angle closure glaucoma. To date, it has been reported after various procedures including filtering surgery, extra and intra capsular cataract surgery, phakic intraocular lens implantation and corneal transplantation even in the absence of a prior diagnosis of angle closure. ${ }^{1,4}$ Other risk factors include an 
axial length of $22.5 \mathrm{~mm}$ or less, narrow irido-corneal angle, female sex, and older age. ${ }^{2,5} \mathrm{~A}$ handful of reports of misdirection following laser procedures such as laser suture lysis, iridotomy, and capsulotomy also appear in the literature. To our knowledge, this appears to be the fourth published case of misdirection following $\mathrm{Nd}$ :YAG capsulotomy. 3,6 We believe that a subclinical picture of aqueous misdirection was developing after cataract surgery, and ultimately this was exacerbated by laser treatment.

Although misdirection typically occurs in the acute post-operative period, there have been reports of cases occurring several weeks to as far out as sixteen years after the inciting surgical procedure. ${ }^{7}$ In this case, we observed progressive AC shallowing and initially unexplained myopic shift occurring six months after uncomplicated phacoemulsification surgery accelerating in the two week postcapsulotomy time frame. Increased myopia has been reported as the first symptom noticed by patients in cases of chronic aqueous misdirection. ${ }^{7}$ The myopic shift can be explained by anterior displacement of the lens to iris diaphragm secondary to increased posterior pressure.

Classic features of aqueous misdirection include central AC shallowing, normal or elevated IOP, and absence of posterior segment pathology. A flat central and peripheral $A C$ is a defining clinical feature, however, IOP may be quite variable. Normal IOP of $\leq 22 \mathrm{~mm} \mathrm{Hg}$ has been observed in $21-50 \%$ of patients, and IOP is more likely to be normal in the setting of a functional glaucoma surgery such as a trabeculectomy or aqueous tube shunt. Therefore IOP is not a reliable indicator of disease. ${ }^{1}$

Our patient had several risk factors for misdirection including female gender, short axial length, and recent anterior segment surgery. The patient presented with normal IOPs at each visit despite a shallowed AC and closed angle. The explanation for normal IOPs in this patient is not entirely clear, however, we believe this was possible due to the presence of some, however limited, trabecular outflow.

Since aqueous misdirection is a diagnosis of exclusion, other causes of acute post-operative AC shallowing such as pupillary block, choroidal hemorrhage, or serous effusion must first be excluded. ${ }^{4}$ We were able to exclude pupillary block by a slit lamp exam that revealed no posterior synechiae and a posterior chamber intraocular lens well placed within the capsular bag. A normal posterior segment exam excluded a serous or hemorrhagic choroidal process.

The pathophysiology of aqueous misdirection is complex and multifactorial. Although the theories regarding how misdirection is initiated are quite diverse, they are all unified by the central concept of an alteration of aqueous flow at the level of the ciliary processes, anterior hyaloid face, zonules, and vitreous body leading to impaired anterior migration of aqueous humor and increased vitreous pressure. It has been demonstrated that the vitreous body becomes less permeable under conditions of high pressure resulting in decreased transvitreal flow. ${ }^{1}$ The increased posterior pressure forces anterior movement of the irido-lenticular diaphragm which is observed clinically as AC shallowing and angle closure., ${ }^{3,6}$

The patient underwent Nd:YAG anterior hyaloidotomy with careful attention to 
treat the anterior hyaloid face. She was also medically managed with one topical aqueous suppressant and a cycloplegic agent. The patient's AC deepened to a normal configuration and myopic shift resolved approximately one year after diagnosis, during which time she underwent laser hyaloidotomy and treatment with medical therapy. Disruption of the anterior hyaloid face with laser can sometimes be an effective strategy for treating aqueous misdirection and avoiding more invasive surgery. However, laser treatment and medical management are often only temporizing measures. The definitive treatment is pars plana vitrectomy combined with iridectomy, zonulectomy, and hyaloidectomy. ${ }^{9}$ Only one of the earlier case reports of misdirection following laser capsulotomy was successfully managed with medical therapy alone; the two other cases required surgical intervention.

We observed a recurrence of clinical signs upon cessation of topical therapy and resolution after restarting, which provided further evidence of the diagnosis of aqueous misdirection in our patient. As of her most recent exam she remains on an aqueous suppressant and a cycloplegic agent. Her AC has deepened to $3.47 \mathrm{~mm}$ which is only 0.18 shallower than her non-affected eye and refraction has improved and stabilized at near plano. She defers surgical intervention at this time.

This case is now the fourth published report of aqueous misdirection after laser capsulotomy. Our patient presented with symptoms of myopic shift and AC shallowing in the absence of an acute IOP elevation. The patient was also able to be successfully managed with medical and laser therapy alone. These are subtle features ophthalmologists should be aware of when following patients in the long term post-operative period. UBM was critical in making the appropriate diagnosis and plan of management.

\section{References}

1. Ramulu PY, Gedde SJ. Aqueous Misdirection. In: Shaarawy TM, Sherwood MB, Hitchings RA, Crowston JG, eds, Glaucoma: Surgical Management. Vol 2. 1st ed. Saunders, 2009; 211-221.

2. Salmon JF. Glaucoma Secondary to Anterior Segment Surgery. In: Albert DM, Miller JW, Azar DT, Blodi BA, Cohan JE, eds, Albert \& Jakobiec's Principles and Practice of Ophthalmology. 3rd ed. Saunders, 2008; 2671-2674.

3. Alvi NP, Cantor LB. Aqueous Misdirection Syndrome. In: Yanoff M, Duker JS, eds, Ophthalmology. 4th ed. Saunders, 2014; 1092-1093.

4. Dave P, Senthil S, Rao HL, Garudadri CS. Treatment Outcomes in Malignant Glaucoma. Ophthalmology 2013; 120(5):984-990.

5. Wollensak J, Pham DT, Anders N. Ciliolenticular block as a late complication in pseudophakia. Ophthalmologe 1995; 92(3):280-283

6. Pham DT, Wollensak J. Ciliary (Malignant) Glaucoma Following Posterior Chamber Lens Implantation. Ophthalmic Surgery 1987; 18(10):741-744.

7. Reed JE, Thomas JV, Lytle RA, Simmons RJ. Malignant Glaucoma Induced by and Intraocular Lens. Ophthalmic Surgery 1990; 21(3):177-180.

8. Hanish SJ, Lamberg RL, Gordon JM. Malignant Glaucoma Following Cataract Extraction and Intraocular Lens Implant. Ophthalmic Surgery 1982; 13(9):713-714.

9. Halkias A, Magauran DM, Joyce M. Ciliary block (malignant) glaucoma after cataract extraction with lens implant treated with YAG laser capsulotomy and anterior hyaloidotomy. British Journal of Ophthalmology 1992; 76:569-570. 
10. Tomey KF, Senft SH, Antonios SR, Shammas IV, Shihab ZM, Traverso CE. Aqueous Misdirection of Flat Chamber After Posterior Chamber Implants With and Without Trabeculectomy. Arch Ophthalmology 1987; 105:770-773.

11. Arya SK, Sonika, Kochmar S, Kumar S, Kang M, Sood S. Malignant Glaucoma as a Complication of Nd:YAG Laser Posterior Capsulotomy. Ophthalmic Surgery, Lasers \& Imaging 2004; 35(3):248-250.

12. Grazyna M, Joanna S. Malignant glaucoma and Central Retinal Vein Occlusion after Nd:YAG laser posterior capsulotomy. Klinika Oezna 2011; 113:7-9.

13. Mastropasqua L, Ciancaglini M, Carpineto P, Lobefalo L, Gallenga PE. Aqueous misdirection syndrome: A complication of neodymium:YAG posterior capsulotomy. J Cataract and Refractive Surgery 1994; 20:563-565.

14. Quigley HA, Friedman DS, Congdon NG. Possible Mechanisms of Primary Angle-Closure and Malignant Glaucoma 2003; 12(2):167-180. 